\title{
Counterpoint: Intraperitoneal Chemotherapy: An Investigational Treatment in Ovarian Cancer
}

\author{
Robert F. Ozols, MD, PhD, Philadelphia Pennsylvania
}

\author{
Key Words \\ Ovarian cancer, intraperitoneal chemotherapy, carboplatin plus \\ paclitaxel, consolidation therapy
}

\begin{abstract}
Intraperitoneal (IP) chemotherapy in ovarian cancer has been studied since 1978. Numerous phase II trials have been performed, which have shown that higher levels can be obtained in the peritoneal cavity compared with systemic circulation after administration of cytoxic agents in a large volume via a semi-permanent catheter. Three randomized trials have been performed in patients with ovarian cancer comparing different IP regimens to standard therapy with intravenous agents. The last two trials from the Gynecologic Oncology Group (GOG) and the Southwest Oncology Group (SWOG) compared two different IP regimens versus standard therapy with intravenous cisplatin plus paclitaxel. Although an improvement in progression-free survival was reported for the IP regimens, they have been associated with unacceptable toxicity, and no IP regimen can be considered standard therapy. Maintenance therapy with IP cisplatin also failed to improve survival in patients who obtained complete remission after intravenous chemotherapy. The GOG is considering another phase III trial of IP therapy that will compare a carboplatin-based regimen versus standard therapy with intravenous paclitaxel plus carboplatin. Unless such a trial shows an improvement in clinical outcome, intravenous carboplatin plus paclitaxel remains the standard of care and IP chemotherapy should not be used outside of a clinical trial. (JNCCN 2004;2:555-560)
\end{abstract}

The Journal of Clinical Oncology has published a series of editorials on the merits of intraperitoneal (IP) chemotherapy

From the Department of Medical Science, Fox Chase Cancer Center Philadelphia, Pennsylvania.

Received August 6, 2004; accepted for publication September 9, 2004.

The author has no financial interest, arrangement, or affiliations with the manufacturers of any products discussed in the article or with their competitors.

Correspondence: Robert F. Ozols, MD, PhD, Senior Vice President, Medical Science, 333 Cottman Avenue, Fox Chase Cancer Center, Philadelphia, PA 19111. E-mail: Robert.Ozols@fccc.edu in ovarian cancer, ${ }^{1-3}$ usually accompanying a clinical trial of this modality. The most recent editorial, by Alberts et al., ${ }^{1}$ concluded with the following paragraph:

"We cannot think of any other setting in oncology where the results of three positive phase III trials have not led to widespread adoption of this superior therapy. The time has come for IP chemotherapy to move beyond the setting of clinical trials and into the standard treatment armamentarium for women with optimally debulked, stage III ovarian cancer. We owe our patients nothing less."

This extraordinary conclusion has somewhat changed the debate on the merits of IP chemotherapy from a dispassionate analysis of data (which certainly permits differing interpretations) to an emotional polemic staking out a position that IP therapy is "standard treatment." It suggests that oncologists who do not use IP therapy, for whatever reason, fail to understand that the phase III trials of IP therapy have shown it to be "superior therapy." Furthermore, it also implies that these same oncologists who do not use IP therapy are somehow depriving their patients inasmuch as they should receive "nothing less" than IP therapy.

Oncologists are duty-bound to provide the best care for their patients. There is no contradiction, as implied by the authors of this editorial, wherein oncologists have used results of phase III trials to change practice patterns in other diseases, but yet "three positive phase III trials" (of IP therapy) "have not led to widespread adoption of this superior therapy." The reason is obvious. Well-trained oncologists and clinical researchers who have reviewed the results of all of the phase III trials do not agree with the contention that these trials are either "positive" or "superior" and certainly do not share the belief that they are "standard treatment." In fact, the Gynecologic Oncology 
Group (GOG) that sponsored all these phase III trials ${ }^{4-6}$ also failed to accept the conclusion that IP therapy is "standard treatment." Although it is possible, and perhaps probable, that the GOG will do yet another trial of IP therapy in ovarian cancer, there is absolutely no question that the control arm must be IV chemotherapy. Moreover, if general agreement was reached with the statement that the results support "IP chemotherapy to move beyond the setting of clinical trials," then performing another randomized trial in which the control arm was IV therapy would be unethical. Legitimate debate remains as to whether these phase III trials should lead to yet another randomized trial of IP therapy, but there clearly is no debate that if such a trial is to be done, it requires a control arm of IV chemotherapy. The experimental arm must be designed to overcome the flaws of the previous studies to unequivocally answer the question of whether the IP route of administration provides a clinically significant advantage in patients with optimally debulked stage III ovarian cancer. Without the results of such a trial, most oncologists rightfully will not routinely administer IP chemotherapy to patients with ovarian cancer for the simple reason that the data generated on the role of IP therapy over the past 25 years do not establish this as a "superior therapy." What then is the controversy regarding the interpretation of these trials?

\section{Rationale for IP Therapy}

The rationale for IP therapy dates back to 1978 and is based on pharmacologic modelling studies performed by Dedrick et al. ${ }^{7}$ that led to the initial clinical trials at the National Cancer Institute (NCI). The administration of cytotoxic agents via a catheter in a large volume leads to a "pharmacologic advantage," defined as the ratio of drug levels in the peritoneal cavity versus the systemic circulation. Numerous pharmacologic studies have confirmed a pharmacologic advantage to using this definition when numerous chemotherapeutic agents are administered into the peritoneal cavity. ${ }^{8}$ What is not clear is whether this pharmacologic advantage leads to greater concentrations of drug directly into the tumor. No experimental evidence is available to support the position that repeated administration of drugs into the peritoneal cavity "literally strips away cancer cells layer by layer with each cycle of therapy (i.e., onion skinning effect)."
Problems with nonuniform drug distribution secondary to adhesions and poor drug penetration of tumor nodules may limit any benefit from simply having higher drug levels in the peritoneal fluid.

Furthermore, most patients with advanced ovarian cancer have retroperitoneal nodal involvement, and it is unclear that there is any advantage from the administration of drugs into the intraperitoneal cavity in patients who already have extraperitoneal disease. It is essential that clinical trials be carefully performed to determine whether, in fact, the IP administration of cytotoxic agents leads to a clinical benefit, defined as prolongation of survival, or, if no survival advantage is found, less toxicity than IV therapy. There is no argument that current IP therapy is associated with increased toxicity compared with IV administration of paclitaxel and carboplatin (by far the most widely used regimen in ovarian cancer and the control arm of all clinical trials currently ongoing in previously untreated patients with advanced disease). Consequently, the major reason that IP chemotherapy has not yet been accepted as "standard therapy" is there is no randomized trial that has shown IP administration of drugs to be superior to IV paclitaxel and carboplatin in patients with optimal stage III ovarian cancer.

Some researchers have argued ${ }^{1}$ that previous randomized trials of IP cisplatin regimens, in which the control arm has been IV cisplatin and paclitaxel,, 5 have shown "positive results." Consequently, one should make the leap of faith that IP therapy with a carboplatin-based regimen would also be superior to IV carboplatin plus paclitaxel. However, these clinical trials have not shown that the IP route of administration was the sole factor in the improvements in progression-free survival and survival in the experimental arms of these trials that included IP therapy. Furthermore, as will be discussed, the toxicity of the experimental arms that included IP administration was of such degree that the authors themselves concluded the regimens could not be recommended for continued development. In addition, the pharmacology of IP carboplatin is different from cisplatin, and initial results suggest that IP carboplatin may be inferior to IP cisplatin. ${ }^{9}$ Consequently, it is difficult to understand how advocates of IP therapy can make the assertion that it should be moved "into the standard treatment armamentarium" but provide no guidance as to which specific regimen should be used. Although 
IP Chemotherapy for Ovarian Cancer

IP carboplatin and paclitaxel may have much less toxicity than observed with previous IP regimens, no evidence is available that they would be clinically superior to the IV administration of these drugs. Consequently, IV treatment with these agents will remain the current standard treatment.

\section{Randomized Trials of IP Therapy}

The GOG and the Southwest Oncology Group (SWOG) have performed three large randomized trials in the past decade in patients with optimal stage III disease (Table 1). In each of these trials, the control arm has been six cycles of IV paclitaxel (135 $\mathrm{mg} / \mathrm{m}^{2}$ ) over 24 hours followed by IV cisplatin (75 $\left.\mathrm{mg} / \mathrm{m}^{2}\right)$ on day 2 . In the first trial, ${ }^{4}$ the experimental arm consisted of IV carboplatin (AUC $=9)$ every 21 days for two courses, then IV paclitaxel $\left(135 \mathrm{mg} / \mathrm{m}^{2}\right)$ over 24 hours followed by IP cisplatin $\left(100 \mathrm{mg} / \mathrm{m}^{2}\right)$ every 3 weeks for six courses. Progression-free survival was superior for patients randomized to the experimental treatment arm (median, 28 vs. 22 months; relative risk, $0.78 ; P=.01$ ). A borderline improvement in overall survival was associated with this regimen (median, 63 vs. 52 months; relative risk $0.81 ; P=.05$ ). There was significantly more toxicity in the experimental arm in regard to neutropenia, thrombocytopenia, and gastrointestinal and metabolic toxicities. Almost one-fifth of the patients received less than two courses of IP therapy. Markman et al. ${ }^{4}$ concluded that because the improvement in overall survival "was of borderline statistical significance and toxicity was greater, the experimental arm was not recommended for routine use." Yet now the results of this trial are used to support the position that IP therapy should be "standard." If it cannot be recommended as "routine," how then can it be recommended that it be "standard?"

Subsequently, Armstrong et al. ${ }^{5}$ reported on results of GOG 172. In this trial, the experimental arm consisted of IV paclitaxel $\left(135 \mathrm{mg} / \mathrm{m}^{2}\right)$ over 24 hours, followed by IP cisplatin $\left(100 \mathrm{mg} / \mathrm{m}^{2}\right)$ plus IP paclitaxel $\left(60 \mathrm{mg} / \mathrm{m}^{2}\right)$ on day 8 . Relative risk of recurrence was 0.73 in the IP group versus the IV group, and possibly further follow-up may have shown the development of a survival advantage for patients who received the IP therapy. However, significantly more grade 3 and 4 toxicity was seen in patients treated with the IP regimen with regard to leukopenia, thrombocytopenia, gastrointestinal toxicity, renal effects, neurologic toxicity, fatigue, infection, metabolic abnormalities, and pain. Of particular note, $19 \%$ of the patients on the IP arm had grade 3 or 4 neurologic toxicity compared with $8 \%$ with standard IV treatment. In addition, the catheter problems were substantial. A recent analysis of the technical problems of IP therapy in this protocol has been presented by Walker et al. ${ }^{10}$ Of 206 patients on the IP arm, 86 (42\%) did not complete all six cycles of therapy. Of these, 34 experienced toxicity, catheter toxicity (primarily abdominal pain), and 52 of the 86 discontinued IP therapy because of catheter complications (primarily infected ports). These complications were reported by GOG investigators, and an even higher complication rate would probably be observed if IP therapy was administered by community oncologists, who have less experience in catheter use and treat smaller numbers of patients with ovarian cancer.

GOG 158 was the last published randomized trial of the GOG in patients with optimal stage III disease. ${ }^{11}$

\begin{tabular}{|c|c|c|c|c|}
\hline Study & Standard Therapy Arm & Experimental Therapy Arm & PFS & OS \\
\hline Markman et al. ${ }^{4}$ & $\begin{array}{l}\text { IV paclitaxel }\left(135 \mathrm{mg} / \mathrm{m}^{2}\right) \\
+ \text { IV cisplatin } 24 \mathrm{~h}\left(75 \mathrm{mg} / \mathrm{m}^{2}\right)\end{array}$ & $\begin{array}{l}\text { IV carboplatin }(\text { AUC }=9) \times 2 \\
\text { followed by } 6 \text { cycles of IV } \\
\text { paclitaxel }\left(135 \mathrm{mg} / \mathrm{m}^{2}-24 \mathrm{~h}\right) \\
\text { plus IP cisplatin }\left(100 \mathrm{mg} / \mathrm{m}^{2}\right)\end{array}$ & 28 vs. $22 \mathrm{mo}$ & 63 vs. $52 \mathrm{mo}$ \\
\hline Armstrong et al. ${ }^{5}$ & Same & $\begin{array}{l}\text { IV paclitaxel }\left(135 \mathrm{mg} / \mathrm{m}^{2}-24 \mathrm{~h}\right) \\
\text { plus IP cisplatin }\left(100 \mathrm{mg} / \mathrm{m}^{2}\right) \text { plus } \\
\text { IP paclitaxel }\left(60 \mathrm{mg} / \mathrm{m}^{2}\right) \text { on day } 8\end{array}$ & RR 0.73 & NA \\
\hline Alberts et al. ${ }^{6}$ & Same & $\begin{array}{l}\text { IV paclitaxel }\left(175 \mathrm{mg} / \mathrm{m}^{2}-3 \mathrm{~h}\right) \\
\text { plus IV carboplatin }(\mathrm{AUC}=7.5)\end{array}$ & 20.7 vs. $19.4 \mathrm{mo}$ & 57.4 vs. $48.7 \mathrm{mo}$ \\
\hline
\end{tabular}

Abbreviations: GOG, Gynecology Oncology Group; SWOG, Southwest Oncology Group, IV, intravenous; IP, intraperitoneal; PFS, progression-free survival; OS, overall survival; AUC, area-under-the-curve; mo, month; NA, not available. 
In this study, the experimental arm was IV paclitaxel $\left(175 \mathrm{mg} / \mathrm{m}^{2}\right)$ in a 3-hour infusion plus IV carboplatin $(A U C=7.5)$. In contrast to the other studies, gastrointestinal, renal, and metabolic toxicities were less in the experimental arm than with intravenous cisplatin and paclitaxel. Neurologic toxicity was similar in both treatment regimens. The relative risk of progression for the carboplatin plus paclitaxel group was $0.88,95 \%$ confidence interval (CI) 0.75 to 1.03 , and the relative risk of death was 0.84 (95\% CI, 0.70-1.05). The results of this trial showing the superiority of carboplatin and paclitaxel compared with cisplatin and paclitaxel were confirmed in another large randomized trial by the AGO. ${ }^{12}$

In these three studies, the experimental arm was associated with an improvement in progression-free survival. Researchers have argued that in the initial two studies this improvement was caused by the IP route of administration. ${ }^{1}$ However, the IP route was not the only variable in the experimental arms. For example, in the Markman et al. study, ${ }^{4}$ all patients received two cycles of high-dose carboplatin as well as an increase in the total number of cycles administered compared with the control arm of six cycles of standard-dose cisplatin plus paclitaxel. In the Armstrong et al. study, ${ }^{5}$ patients received high doses of IP cisplatin $\left(100 \mathrm{mg} / \mathrm{m}^{2}\right)$ as well as IP paclitaxel on day 8 . Consequently, the effect of the dose of cisplatin and the schedule (day 1, day 8) of paclitaxel contributed to the toxicity and may have also had an effect on progression-free survival. In fact, the experimental arms of all three studies have in common higher doses of platinum equivalents than the control arm. Early retrospective studies have suggested that dose intensity of platinum compounds is important in ovarian cancer. ${ }^{13}$ Although a GOG prospective randomized trial of two different dose intensities of cisplatin in suboptimal stage III and IV patients failed to show a clinically significant effect of dose intensity on outcome, ${ }^{14}$ no trial of dose intensity of platinum compounds has been performed in optimal stage III patients. Consequently, it is impossible to exclude the possibility that one of the reasons associated with improved outcome in these three trials in patients with optimal stage III disease is the dose of the platinum compounds.

Researchers have suggested that the unacceptable toxicity seen in GOG 172 could be ameliorated by several modifications that would not impact the improvements seen in progression-free survival.
Researchers at the Arizona Cancer Center claimed that a port-a-cath designed for IV injection "virtually eradicates fibrosheath formation and greatly diminishes the risk of small bowel obstruction and perforation." Some researchers have also postulated that the toxicity could be markedly decreased by dropping the dose of cisplatin or completely eliminating cisplatin from the IP route and replacing it with carboplatin. ${ }^{1}$ These modifications of the IP regimen may decrease toxicity; however, simply making these modifications without confirming their impact on clinical outcome, which can only be ascertained through a prospective randomized trial in which the control arm would be IV carboplatin and paclitaxel, would be unwise.

The difficulties in performing randomized trials with IP chemotherapy cannot be underestimated. Piccart et al. ${ }^{15}$ recently reported the results of an 8-year randomized phase III study from the EORTC in which ovarian cancer patients with a pathologically complete remission were randomized to receive IP chemotherapy or no further therapy. Overall survival was the primary endpoint, and progression-free survival was the secondary endpoint. However, from 1988 to 1997 , only 153 patients in a pathologically confirmed complete remission after platinum-based IV therapy were randomized to four courses of IP cisplatin $\left(90 \mathrm{mg} / \mathrm{m}^{2}\right)$ every 2 weeks (or observation). After a median follow-up time of 8 years, 80 patients (52\%) had progressed with difference in the pattern to relapse between the two groups, and 75 patients have died. The hazard ratio for PFS and OS was 0.89 $(0.59-1.33)$ and $0.82(0.59-1.29)$, respectively. This study accrued extremely slowly, had to be stopped prematurely, and was plagued with a significant proportion of women who were either ineligible $(10 \%)$ or had major protocol violations (11\%). Researchers postulated that this was probably because of the limited experience with the technical aspects of IP chemotherapy. The authors themselves conclude that the results of this study "do not support a change in clinical practice." 15

\section{IP Carboplatin plus Paclitaxel}

The results of a recent report of first-line IP carboplatinbased chemotherapy in 165 Japanese women with ovarian cancer have recently been reported.${ }^{16}$ In this study, the median progression-free survival of optimal stage III patients $(<2 \mathrm{~cm}$ disease $)$ treated with IP carboplatin 
regimens was 22 months, and median survival was 51 months. Although comparisons across clinical trials cannot be used to draw firm conclusions, it should be noted that PFS and OS are no better, and possibly worse, compared with a similar optimal $(<1 \mathrm{~cm})$ patient population in GOG 158 who were treated using IV carboplatin plus paclitaxel. With the IV regimen, the median PFS was 21 months and median OS was 57 months. This raises questions about whether IP therapy with a carboplatin regimen is even worth exploring in an additional phase III trial. This is a legitimate debate that is ongoing with the GOG. Do the results of the previous two randomized trials plus results of the Fujiwara study ${ }^{16}$ support another large randomized trial in optimal stage III patients? Would other high priority studies have a potentially greater impact than merely substituting IP carboplatin for IV carboplatin?

\section{Conclusions}

More than 25 years have passed since the modern era of IP chemotherapy began with patients receiving highvolume IP drugs administered via a semipermanent Tenckhoff catheter. Numerous phase II trials finally led to three prospective randomized trials comparing IV administration to IP regimens. Although the IP regimens have been associated with an improvement in progression-free survival and borderline improvement in overall survival, the results cannot be translated into standard practice at this time. Investigators themselves noted that the IP regimens are associated with unacceptable toxicity and cannot be considered "routine" therapy. In addition, none of the IP regimens has clearly identified the role of the IP route of administration, and dose and schedule may possibly impact the results reported. In addition, none of the IP regimens has been compared directly against IV carboplatin and IV paclitaxel, which remains the current approach for patients with advanced-stage optimal ovarian cancer. Finally, the toxicity of IP catheters is substantial, and in the last GOG study, more than $40 \%$ of patients were not able to complete all six cycles of IP therapy. ${ }^{10}$ Although it is possible that the toxicity of IP therapy can be ameliorated using different administration techniques and different drugs (i.e., replacing cisplatin and carboplatin), before such regimens can be routinely used in the community, they must be validated in prospective randomized controlled trials. Legitimate debate continues, however, based on the results of these prior studies and on recent reports of IP carboplatinbased chemotherapy in Japanese women with ovarian cancer, ${ }^{16}$ as to whether sufficient clinical benefit could be expected from such an approach, and whether patient resources should be used for yet another large trial comparing IP versus IV chemotherapy. If such a trial is performed and if the results show clinical superiority for the IP route of administration with acceptable toxicity, then and only then can IP therapy be considered "standard" for patients with optimal stage III ovarian cancer.

\section{References}

1. Alberts DS, Markman M, Armstrong D, et al. Intraperitoneal therapy for stage III ovarian cancer: A therapy whose time has come! J Clin Oncol 2002;20:3944-3946.

2. McGuire WP. Intraperitoneal therapy for ovarian cancer: A sacrifice bunt. J Clin Oncol 2001;19:1001-1007.

3. Ozols RF. Intraperitoneal therapy in ovarian cancer: Time's up. J Clin Oncol 1991;9:197-199.

4. Markman M, Bundy BN, Alberts DS, et al. Phase III trial of standard-dose intravenous cisplatin plus paclitaxel versus moderately high-dose carboplatin followed by intravenous paclitaxel and intraperitoneal cisplatin in small-volume stage III ovarian carcinoma: An intergroup study of the Gynecologic Oncology Group. Southwestern Oncology Group, and Eastern Cooperative Oncology Group. J Clin Oncol 2001;19:1001-1007.

5. Armstrong DK, Bundy BN, Baergen R, et al. Randomized phase III study of intravenous (IV) paclitaxel and cisplatin versus IV paclitaxel, intraperitoneal (IP) cisplatin and IP paclitaxel in optimal stage III epithelial ovarian cancer (OC): A Gynecololgic Oncology Group trial (GOG 172) (Abstr \#803). Proc Am Soc Clin Oncol 2002;21:201a.

6. Alberts DS, Liu PY, Hannigan EV, et al. Intraperitoneal cisplatin plus intravenous cyclophosphamide versus intravenous cisplatin plus intravenous cyclophosphamide for stage III ovarian cancer. N Engl J Med 1996;335:1950-1955.

7. Dedrick RL, Myers CE, Bungay PM, et al. Pharmacokinetic rationale for peritoneal drug administration in the treatment of ovarian cancer. Cancer Treat Rep 1978;62:1-11.

8. Markman M. Intraperitoneal chemotherapy. Semin Oncol 1991;18:248-254.

9. Markman M, Reichman B, Hakes T, et al. Evidence supporting the superiority of intraperitoneal cisplatin compared to intraperitoneal carboplatin for salvage therapy of smallvolume residual ovarian cancer. Gynecologic Oncology 1993;50:100-104.

10. Walker JL, Armstrong D, Bundy BN, et al. Intraperitoneal catheter outcomes on GOG 172: Randomized trial of IV vs. IP chemotherapy in optimal ovarian cancer (Abstrt \#20). 35th Annual Meeting Society of Gynecologic Oncologists 2004:84.

11. Ozols RF, Bundy BN, Greer BE, et al. Phase III trial of carboplatin and paclitaxel compared with cisplatin and paclitaxel 
Ozols

in patients with optimally resected stage III ovarian cancer: A Gynecologic Oncology Group Study. J Clin Oncol 2003;21:3194-3200.

12. du Bois A, Luck H-J, Meier W, et al. A randomized clinical trial of cisplatin/paclitaxel versus carboplatin/paclitaxel as first-line treatment of ovarian cancer. J Natl Cancer Inst 2003;95:1320-1330.

13. Levin L, Hryniuk WM. Dose intensity analysis of chemotherapy regimens in ovarian carcinoma. J Clin Oncol 1987;5: 756-767.

14. McGuire WP, Hoskins WJ, Brady MF, et al. Assessment of dose-intensive therapy in suboptimally debulked ovarian cancer: A Gynecologic Oncology Group Study. J Clin Oncol 1995;13:1589-1599.

15. Piccart MJ, Floquet $A$, Scarfone G, et al. Intraperitoneal cisplatin versus no further treatment: 8 -year results of EORTC 55875 , a randomized phase III study in ovarian cancer patients with a pathologically complete remission after platinumbased intravenous chemotherapy. Int J Gynecol Cancer 2003;13:196-203.

16. Fujiwara K, Sakuragi N, Suzuki S, et al. First-line intraperitoneal carboplatin-based chemotherapy for 165 patients with epithelial ovarian carcinoma: Results of long-term followup. Gynecol Oncol 2003;90:637-643. 